\title{
Fortalecimiento de las competencias de interpretación y solución de problemas mediante un entorno virtual de aprendizaje
}

\author{
Strengthening of interpretation skills and problem solving through a virtual \\ learning environment
}

César Augusto Gutiérrez-Rodríguez'

Recibido: agosto 26 de 2017

Aceptado: diciembre 28 de 2017

\begin{abstract}
Resumen
Este artículo es resultado de una investigación aplicada cuasiexperimental, cuyo objetivo fue fortalecer la competencia de interpretación y solución de problemas desde la asignatura de tecnología e informática, integrando para ello las Tecnologías de la Información y la Comunicación, TIC, en el proceso enseñanza aprendizaje. El trabajo se adelantó con 30 estudiantes de grado décimo en una Institución Educativa de Cali, Colombia. Se utilizó un Entorno Virtual de Aprendizaje, EVA, diseñado bajo las orientaciones de la técnica didáctica del Aprendizaje Basado en Problemas, ABP. La caracterización inicial de la competencia se realizó a partir del resultado de las pruebas Saber $11^{\circ} 2015$, junto con una evaluación diagnóstica diseñada con preguntas del cuadernillo de pruebas Saber de grado noveno. Luego de implementar el EVA, los resultados de la prueba final indican que el grupo mejoró la competencia de interpretación y solución de problemas, cuando se compara su desempeño con respecto a la prueba inicial. Igualmente, se observaron cambios positivos en los ámbitos social y comportamental, lo que indica que la mediación tecnológica produjo un mayor compromiso de los estudiantes en su proceso formativo.
\end{abstract}

Palabras clave: entorno virtual de aprendizaje, aprendizaje basado en problemas, educación virtual, solución de problemas.

\begin{abstract}
This article is the result of an applied quasi-experimental research, whose objective was to strengthen the competence of interpretation and problem solving from the subject of Technology and Information Technology, integrating the Information and Communication Technologies, ICT, in the teaching-learning process. The work started with 30 tenth grade students in an Educational Institution of Cali, Colombia. We used a Virtual Learning Environment, VLE, designed under the guidance of the didactic technique of Problem Based Learning (PBL). The initial characterization of the competition was made based on the result of the Saber 11/2015 tests, together with a diagnostic evaluation designed with questions from the Saber 9th grade test booklet. After implementing the VLE, the results of the final test indicate that the group improved the interpretation and problem solving competence, when comparing its performance with respect to the initial test. Likewise, positive changes were observed in the social and behavioral fields, which indicate that technological mediation produced a greater commitment of the students in their training process.
\end{abstract}

Keywords: virtual learning environment, problem based learning, virtual learning, problem.

1 Ingeniero de sistemas y telecomunicaciones, Magíster en informática educativa, Escuela Militar de Aviación Marco Fidel Suárez, Cali, Colombia. E-mail: profecesar15@gmail.com 


\section{Introducción}

En la actualidad, el modelo de formación por competencias, entendidas como el conjunto de conocimientos, habilidades, destrezas y valores que adquiere un estudiante, constituye la base del sistema educativo colombiano (García-Quiroga, Coronado \& Giraldo-Ospina, 2017). El Ministerio de Educación Nacional, MEN, ha establecido los estándares básicos de competencia para las áreas obligatorias, salvo la de tecnología e informática, en los niveles de educación básica y media (MEN, 2006). Entre las competencias más importantes se encuentra la de interpretación y solución de problemas, en la cual el estudiante debe evidenciar capacidad para formular problemas a partir de diferentes situaciones, aplicar diversas estrategias y justificar la elección de los métodos de solución (Hernández-Suárez, Prada-Núñez \& Gamboa-Suárez, 2017; Barrera-Mesa, Fernández-Morales \& Duarte, 2017a).

La educación en Colombia, particularmente en la ciudad de Santiago de Cali, presenta una serie de dificultades relacionadas, entre otras, con la práctica misma de la enseñanza (Jaramillo-Arcila \& Quintero-Calvache, 2014). Para los profesores cada una de sus clases representa un reto en el propósito de atraer la atención de sus estudiantes, independientemente del nivel o área de formación (Aldana-Bermúdez \& López-Mesa, 2016; Bernal-Pinzón, 2017). Esto resulta aún más complejo ya que las características de una época tecnológica, con una sobreoferta de medios audiovisuales como la televisión, el cine e internet, hace de dichos estudiantes individuos curiosos y muy dinámicos, que difícilmente se adaptan a los modelos pedagógicos tradicionales (Buitrago-Guzmán, 2014; Parada-Hernández \& Suárez-Aguilar, 2014).

En este sentido, las Tecnologías de la Información y la Comunicación, TIC, han surgido como una herramienta con gran potencial para la mediación pedagógica (Quiroga-Parra, Torrent-Sellens \& Murcia-Zorrilla, 2017). No obstante, un aspecto que ha limitado la integración de las TIC en la educación es considerar los dispositivos tecnológicos como un obstáculo para el proceso educativo (García-Amaya, Fernández-Morales \& Duarte, 2017), si bien los dispositivos pueden presentarse como distractores, usualmente el beneficio de su empleo es grande, pues suelen desarrollar actitudes más positivas, mayor confianza en el uso fundamentado de los mismos y una actitud crítica que se refleja en el cambio de algunas de sus concepciones y prácticas (Valencia-Serrano \& Caicedo-Tamayo, 2017; Avella-lbáñez, Sandoval-Valero \& Montañez-Torres, 2017).

Esto último se refleja en diversas experiencias a partir de Entornos Virtuales de Aprendizaje, EVA. Entre ellas se destacan el trabajo de Jaramillo-Arcila y Quintero-Calvache (2014), el cual promueve el pensamiento aleatorio en estudiantes de los grados cuarto y quinto de primaria, en una Institución Educativa, IE, de Santiago de Cali. Igualmente, se tiene el estudio de Rico-González (2011), que aplicó exitosamente un ambiente virtual para la enseñanza de física en el nivel de educación media. Otro trabajo es el de Aguirre (2015), respecto a una experiencia educativa nacional en el Salvador empleando el programa de diseño de videos Kodu para desarrollar habilidades computacionales. Asimismo, Barrera-Mesa, Fernández-Morales y Duarte (2017b), reportan un ambiente de aprendizaje que integra diversas herramientas TIC para facilitar el aprendizaje de los operadores mecánicos en estudiantes de grado séptimo, con resultados positivos en cuanto a la apropiación de conocimiento.

En la IE Santo Tomás, de Santiago de Cali, en el año 2015 los estudiantes de grado once, presentaban promedios bajos en el área de matemáticas, destacando la competencia de razonamiento y argumentación en un nivel básico y la de planteamiento y resolución de problemas en un nivel insuficiente. Igualmente, la Subsecretaría de Desarrollo Pedagógico del municipio indicó que la mayor parte del problema radicaba en las prácticas de aula utilizadas por los docentes (Rosas, 2015). 
Este artículo presenta el desarrollo, implementación y evaluación de un EVA, con el propósito de fortalecer el proceso de enseñanza-aprendizaje, especialmente en cuanto a la competencia de interpretación y solución de problemas, bajo la técnica didáctica de Aprendizaje Basado en Problemas, ABP. El trabajo se desarrolló con los estudiantes de grado décimo de la sede CASD, en la IE Santo Tomás, de la ciudad de Cali.

\section{Marco teórico y metodología}

\subsection{Referente teórico}

La investigación asumió el Entorno Virtual de Aprendizaje, EVA, como una herramienta didáctica en el aula, con el ánimo de estimular la competencia de interpretación y solución de problemas. Igualmente, se consideró como técnica didáctica al Aprendizaje Basado en Problemas, ABP. A continuación, se hace una breve presentación de estos conceptos.

Según Díaz, citado por Blanco-Sánchez (2012), los recursos didácticos son todo el conjunto de elementos o estrategias que se utilizan o se pueden utilizar por el docente como complemento en su labor diaria. Ejemplo de ellos son los prototipos didácticos, las maquetas, los carteles $y$, más recientemente, los videos, software educativo, juegos didácticos y páginas de internet, entre otros (Castro-Galeano, Pinto-Salamanca \& Amaya-Quitián, 2014; Contreras-Chinchilla \& Escalona-Ustariz, 2014; Valdelamar-Zapata, Ramírez-Cruz, Rodríguez-Rivera \& Morales-Rubiano, 2015; León-Medina \& Torres-Barahona, 2016; Rodríguez-Cepeda, 2016; Ramírez-Hurtado, Gil-Monsalve, Medina-Barreto \& Cruz-Muñóz, 2016).

El ABP es una técnica didáctica enfocada en el aprendizaje, la investigación y la reflexión del estudiante frente a un problema planteado por el docente (Fernández \& Duarte, 2013). En muchas ocasiones el ABP permite que los estudiantes adquieran conocimientos y puedan aplicarlos a la solución de un problema real o ficticio, sin necesi- dad de recurrir a la clase magistral. El $\mathrm{ABP}$ ha sido empleado en todos los niveles de formación y en áreas tan disímiles como ciencias básicas, ingeniería, administración y formación de educadores, por mencionar algunas (Parra-León, Duarte \& Fernández-Morales, 2014; Medina-Rojas, Núñez-Santa, Sánchez-Medina \& Cabrera-Medina, 2017; Almeida-Hidalgo, 2016).

Un Entorno Virtual de Aprendizaje, EVA, es un espacio educativo usualmente disponible en internet, que integra recursos educativos, promueve la interactividad de los estudiantes y brinda actividades de aprendizaje estructuradas (Riveros-Hernández, Nausan-García, García-Miranda \& Palacios-Osma, 2017). El EVA posee una doble dimensión: tecnológica y educativa. La primera se encuentra expresada en las herramientas o aplicaciones informáticas, las cuales sirven de soporte a las propuestas educativas; aunque pueden variar de un EVA a otro, permiten acciones fundamentales como: la publicación de materiales, la interacción entre los miembros del grupo y la organización de la asignatura. La segunda dimensión se refleja en el proceso de enseñanza aprendizaje, y tiene como principal aporte recordar que el EVA se trata de un espacio humano, social y dinámico, fundamentado en la interacción del docente y los estudiantes con el propósito de plantear y resolver actividades didácticas (Salinas, 2011).

El diseño instruccional, DI, según Berger y Kam (2006), es la ruta detallada a seguir para el desarroIlo, evaluación e implementación de situaciones que facilitan el aprendizaje de pequeñas y grandes unidades de contenidos, en diferentes niveles de complejidad. En este proyecto el DI se elaboró según el proceso propuesto por Belloch (2011), de Análisis, Diseño, Desarrollo, Implementación y Evaluación, ADDIE. Inicialmente se genera el producto tecnológico y luego se aplican algunos principios en cuanto a: qué es un EVA, su doble dimensión (tecnológica y educativa), cuáles son los criterios de selección, por qué enseñar con y en Entornos Virtuales y cuál es el papel del docente 
en la utilización de dichas herramientas (Salinas, 2011).

La integración de las TIC en el proceso de enseñanza-aprendizaje, según la propuesta de Mishra y Koehler (2006), se compone de 3 conocimientos fundamentales: i) Contenidos, es la parte donde se deben identificar cuáles son los temas disciplinares que se desarrollarán en el EVA; II) Pedagógico, donde se describen las didácticas y herramientas para planificar la actividad y evaluar a los estudiantes, y iii) Tecnológico: donde se seleccionan los recursos tecnológicos y su aplicación para el alcance de los objetivos, todo enmarcado en un contexto determinado.

Entre las razones para emplear este tipo de herramientas se tienen: el adaptar la enseñanza al contexto sociocultural contemporáneo; la multialfabetización entendida como un aprendizaje que integre las diversas formas y lenguajes de representación y comunicación de la información: textual, icónica, sonora, hipertextual, audiovisual y tridimensional, entre otras, a través de distintas tecnologías; promover la innovación curricular, orientada al mejoramiento de la enseñanza y del aprendizaje (Salinas, 2011). Las TIC también impactan la motivación y concentración del estudiante en el proceso de enseñanza-aprendizaje, por las posibilidades de interacción y animación a la hora de presentar los conceptos y las actividades, lo cual permite aumentar la permanencia de los estudiantes en el aula (Claro, 2010).

\subsection{Metodología}

El presente trabajo corresponde a una investigación aplicada, con un diseño cuasi-experimental, definida por Cook y Campbell (1979), con las siguientes características: i) se busca el estudio del efecto de la variable independiente sobre la variable dependiente de la investigación, persiguiendo el establecimiento de relaciones causales. ii) el investigador no interviene en la formación de los grupos, de manera que recurre a grupos intactos o naturales. iii) se suelen utilizar en investigaciones de carácter aplicado, por lo que se desarrollarán, principalmente, en contextos naturales alejados del laboratorio. El estudio plantea dos variables, una dependiente que es la competencia de interpretación y solución de problemas, la cual sufre cambios por la intervención de una variable independiente, como es la metodología para integrar el EVA al proceso de enseñanza-aprendizaje.

La investigación se desarrolló en la Institución Educativa Santo Tomás de la ciudad de Cali. La institución está conformada por cuatro sedes, las cuales abarcan desde preescolar hasta grado once. La sede escogida fue el Centro Auxiliar de Servicios Docentes, CASD, en la cual se encuentran los grados décimo y once, además recibe estudiantes de otras 19 instituciones para realizar estudios de media técnica.

La población objeto de estudio fueron los 180 estudiantes de grado décimo, conformado por hombres y mujeres entre los 15 y 17 años de edad, que viven en barrios del sector, de estratos 0 a 2 . La muestra donde se aplicó el instrumento de evaluación fue de 30 estudiantes seleccionados al azar.

Las fases de la metodología se llevaron a cabo de acuerdo al modelo de diseño instruccional ADDIE, mencionado en el apartado anterior. Cada etapa se encuentra en plena relación con las otras y da inicio o continuidad a la siguiente; por ejemplo, las metodologías, herramientas y técnicas seleccionadas fueron resultado de la etapa de análisis. En esta etapa es importante reconocer que la prueba Saber evalúa el desarrollo de competencias básicas en los ciclos $3^{\circ}, 5^{\circ},{ }^{\circ}$. Esta prueba se enfoca en evaluar las competencias en las áreas de lenguaje, matemáticas y ciencias naturales, ver tabla 1. En la prueba de Matemáticas se definen tres competencias que recogen los elementos centrales de los procesos de pensamiento, descritos en los Estándares Básicos de Competencias: interpretación y representación, formulación y ejecución, y argumentación. (ICFES, 2016a). 


\begin{tabular}{lll}
\hline Lenguaje & Matemáticas & Ciencias Naturales \\
\hline $\begin{array}{l}\text { Competencia comunicativa a través } \\
\text { de dos procesos: }\end{array}$ & $\begin{array}{l}\text { Razonamiento y argumentación } \\
\text { Lectura }\end{array}$ & $\begin{array}{l}\text { Uso comprensivo del conocimiento } \\
\text { científico } \\
\text { delación }\end{array}$ \\
$\begin{array}{l}\text { Escritura } \\
\text { Planteamiento y resolución de pro- }\end{array}$ & Explicación de fenómenos \\
\hline
\end{tabular}

Tabla 1. Competencias evaluadas en las pruebas Saber (ICFES, 2016b).

En el año 2015 , en las pruebas Saber $11^{\circ}$, la IE Santo Tomás tuvo resultados similares a los promedios municipal y nacional. En la tabla 2 se evidencia que los puntajes en matemáticas y razonamiento cuantitativo, que evalúan la interpretación y so- lución de problemas, tienen resultados que por muy poco pasan la mitad de los puntos evaluados, situación preocupante no solo para la institución educativa, sino también para el país.
Nivel de Reporte Nivel de Reporte

\section{Matemáticas Promedio (Desviación)}

\section{Razonamiento cuantitativo\} (Desviación)}

\begin{tabular}{lll} 
Colombia (8979 establecimientos) & 51.5 & 52.6 \\
Cali & $(9.3)$ & $(10.6)$ \\
(283 establecimientos) & 49.7 & 50.8 \\
& $(9)$ & $(10.3)$ \\
Institución Educativa Santo Tomás & 51.6 & 52.7 \\
& $(8.2)$ & $(9.7)$ \\
\hline
\end{tabular}

Tabla 2. Resultados de la prueba saber $11^{\circ}$, año 2015 (Rosas, 2015).

Adicionalmente, se realizó una prueba diagnóstica con los estudiantes de la muestra, la cual permitió conocer su nivel en la competencia de interpretación y solución de problemas. La prueba consistió en 10 preguntas que se seleccionaron del Cuadernillo de prueba Saber $3^{\circ}, 5^{\circ}$ y $9^{\circ} 2015$, Matemáticas Grado $9^{\circ}$ (ICFES, 2016c). Los ejercicios se alojaron en la página web (www.thatquiz.org), para facilitar su implementación en línea; el puntaje de la prueba corresponde al número de aciertos.

A partir de los resultados de la etapa anterior, se determinó desarrollar un curso que fortaleciera la competencia de interpretación y solución de problemas en los estudiantes de grado décimo. La idea es fomentar el desarrollo de competencias mediante un papel activo de los estudiantes, siendo ellos quienes construyen su propio conocimiento con la guía desarrollada por el docente, en un entorno mediado por TIC. Como herramienta didáctica se optó por diseñar un EVA, teniendo en cuenta sus características interactivas; es decir, se pretende que el conjunto de sus recursos, como: animaciones, chat y foros, entre otros, estimularan la participación del estudiante. El diseño y las actividades propuestas se describen en el siguiente apartado. 
Luego de seleccionar los contenidos del curso y segmentarlos en tres unidades para la creación de los Objetos Virtuales de Aprendizaje, OVA, se desarrolló el EVA propiamente dicho. El entorno se elaboró en la plataforma Moodle, la cual se encuentra alojada en la web y permite crear cursos en línea o, como en el presente caso, Entornos Virtuales (Barrera-Messa et al., 2017b). Esta plataforma contiene dentro de sus posibilidades el uso de herramientas como son: foros, glosario, chat, tarea, certificado, encuesta, lista de verificación, wiki y cuestionarios, entre otras (Astudillo, Sanz \& Willging, 2012).

El grupo piloto utilizó como herramienta didáctica el EVA desarrollado, con el propósito de identificar su impacto en el proceso de enseñanza-aprendizaje, particularmente en la estimulación de la competencia de interpretación y solución de problemas. En cada sesión se realizó un informe de observación en el cual se tomaban en cuenta aspectos comportamentales y sociales, como: la asistencia de los estudiantes, hora de llegada al aula, su participación en el proceso, disposición y el porcentaje de quienes realizaban las actividades propuestas. Al finalizar la implementación del EVA, los mismos 30 estudiantes presentaron una prueba final, similar a la inicial, para medir el nivel alcanzado en la competencia de interpretación y solución de problemas.

\section{Resultados y discusión}

\subsection{Prueba inicial}

En una escala de 0 a 100, en los grupos experimental y control, la mayoría de estudiantes obtuvo puntajes de 60 o menos, e incluso algunos alcanzaron puntajes de 30, 20 y 10. En contraste, solo cuatro obtuvieron 60 puntos, y 1 de ellos 70 puntos, ninguno superó tal cifra. Es decir, de los 30 estudiantes solo cinco "aprobaron" y lo hicieron con el más bajo valor posible, encontrándose alejados de resultados altos o al menos satisfactorios. Lo anterior sugiere que los estudiantes aún no han explorado todas sus capacidades en cuanto al pensamiento lógico y la solución de problemas; tampoco están explotando todas las competencias adquiridas en el trascurso de sus estudios para la comprensión y análisis de problemas y las competencias básicas en matemáticas, con lo cual se lograría una mejoría en el desempeño en las pruebas Saber $11^{\circ}$, principalmente en las áreas de matemáticas y ciencias.

La prueba inicial permitió ratificar las falencias identificadas previamente, comprobando el bajo desarrollo de la competencia de interpretación y solución de problemas de los estudiantes $y$, por ende, la necesidad de fortalecer dicho aspecto. Luego de la prueba, los estudiantes se mostraron dispuestos a participar en desarrollar actividades que les permitieran mejorar su desempeño.

\subsection{El EVA desarrollado}

Los OVA, desarrollados mediante la herramienta de diseño Adobe Captive 9, se integraron al entorno Virtual de Aprendizaje, EVA. Los OVA contienen tres Unidades, al final de las cuales el EVA presenta una evaluación final que corresponde a la misma evaluación diagnóstica. Esto CON EL FIN de medir el impacto de la implementación del producto desarrollado en el grupo piloto sobre la competencia de interpretación y solución de problemas. La unidad de Introducción ofrece una bienvenida a los estudiantes y presenta toda la información del curso, ver figura 1. 
Hola, César..

Te invito a que empieces este maravilloso viaje hacia el conocimiento, explorando cada uno de los botones en el contenido. ¡Adelante!
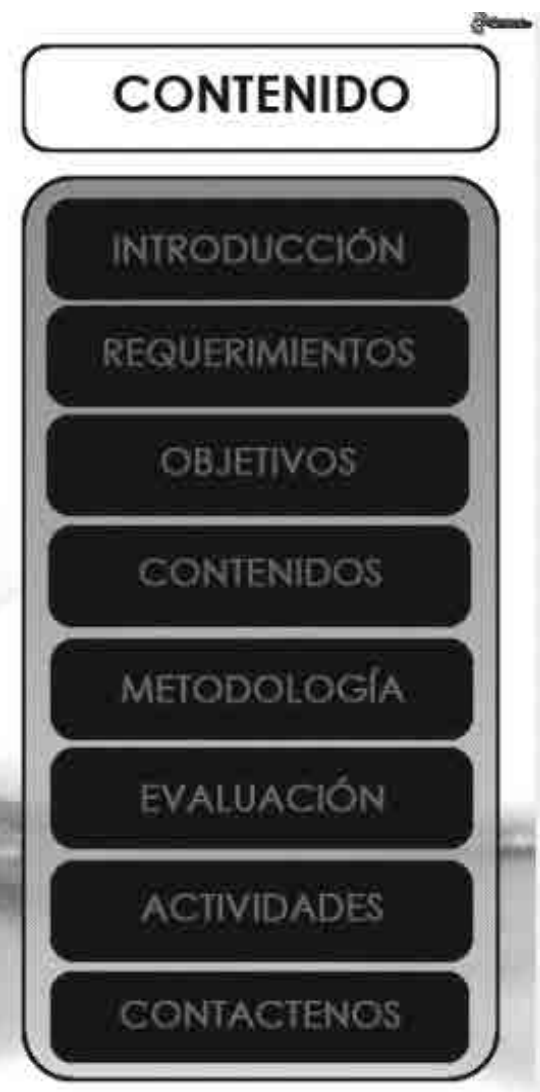

Figura 1. OVA de Introducción.

La primera unidad está dividida en cuatro momentos presentados en forma de videojuego, como se ilustra en la figura 2. El primer momento presenta los conceptos a desarrollar en la unidad, el segundo es una actividad interactiva, el tercero es otra actividad interactiva con mayor grado de complejidad. Al finalizar cada actividad propuesta en los tres primeros momentos, el estudiante deberá presentar una solución en MS-Word y subirlo a un espacio dentro del EVA, donde el docente realiza la respectiva revisión y retroalimentación. El cuarto momento presenta una actividad evaluativa, de tipo sumativo, con un cuestionario que abarca todos los conceptos vistos e interiorizados. 


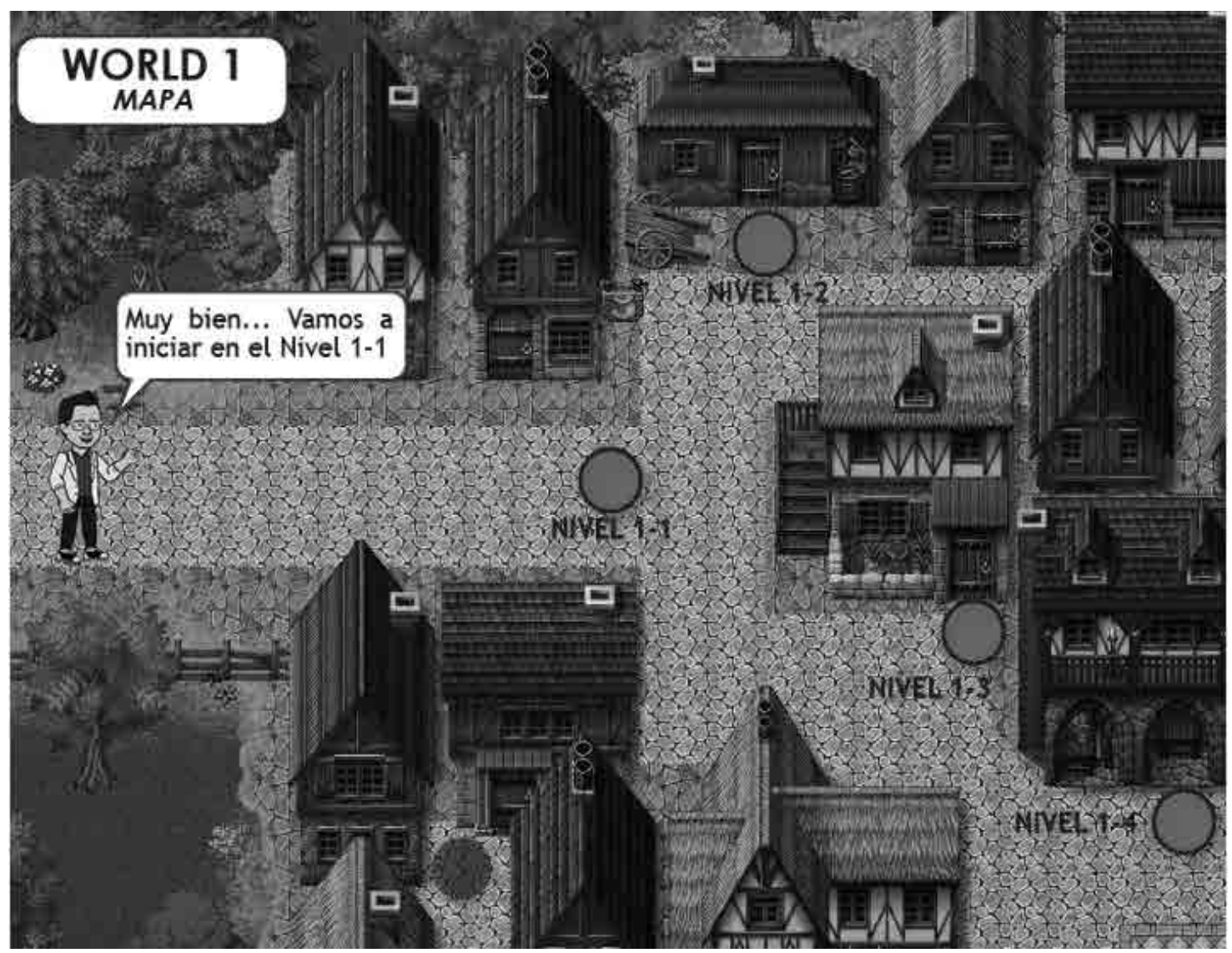

Figura 2. OVA Unidad 1.

La segunda unidad también se divide en 4 momentos, como se ilustra en la figura 3. El primer momento presenta la descripción de los conceptos de la unidad, el segundo y tercer momento presentan un videoquiz que contiene las instrucciones para crear un juego en Scratch, asumiendo un reto adicional planteado por el docente; al finalizar, el resultado del videoquiz deberá ser subido a un espacio asignado en el EVA. El último momento presenta un reto diferente, con un grado más alto de dificultad, pues el estudiante solo contará con un enunciado para desarrollar la actividad, iniciando de cero, a diferencia de los anteriores donde contaba con videos e instrucciones como guía; aAl finalizarlo, deberá subirlo a un espacio asignado en el EVA. Lo que se pretende con la segunda unidad es que los estudiantes no solo sean consumidores de información, sino también creadores de la misma, a la vez que aumentan sus recursos para afrontar y solucionar problemas. 


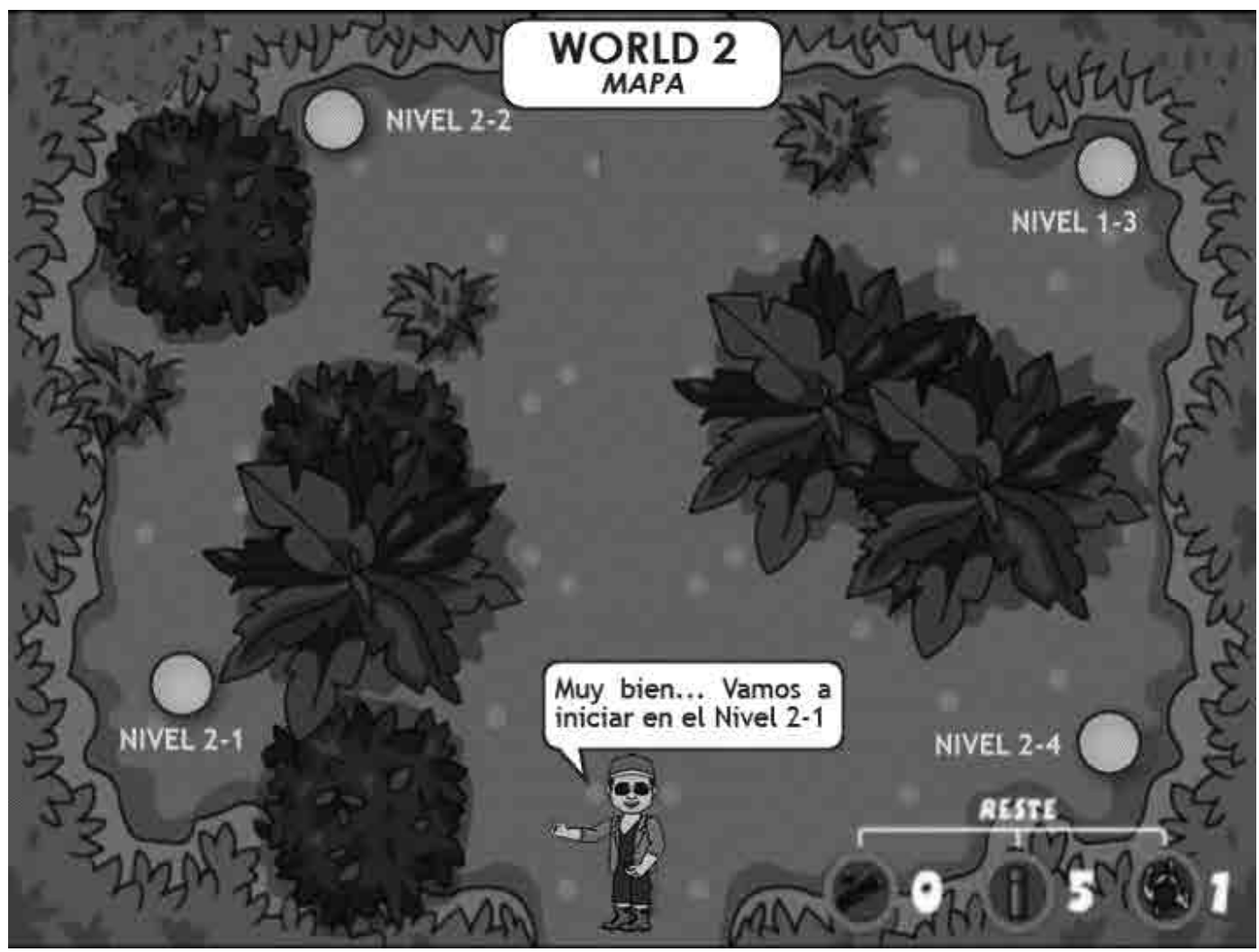

Figura 3. OVA Unidad 2.

El desarrollo del producto tecnológico se llevó a cabo buscando hacer llamativos los OVA de cada unidad, junto con el EVA. Esto mediante el uso de animaciones y manejo de colores vivos, combinado con información clara y precisa que potenciara las técnicas didácticas y herramientas TIC propuestas, como lo sugieren diversos autores para mejorar el efecto de las TIC en el aula (Pinto-Salamanca, Sofroni-Esmeral \& Jiménez, 2015; Alvarez \&Taboada, 2016; salcedo-Ramírez, Fernández-Morales \& Duarte, 2017).

\subsection{Prueba final}

El grupo piloto presentó avances significativos, pues solo 4 de los 15 estudiantes reprobaron con puntajes inferiores a 60 . A su vez, cinco estudiantes se mantuvieron en la barrera de los 60 puntos, y si bien "aprobaron", presentan un nivel regular. Además, 6 estudiantes alcanzaron puntajes óptimos, 3 de ellos con 70 y los otros 3 con 80 puntos, lo cual, aunque no se manifiesta como una tendencia absoluta, expone un patrón de desempeño favorable. La figura 4 presenta la comparación de los resultados obtenidos en las pruebas diagnóstica y final para el grupo piloto. 


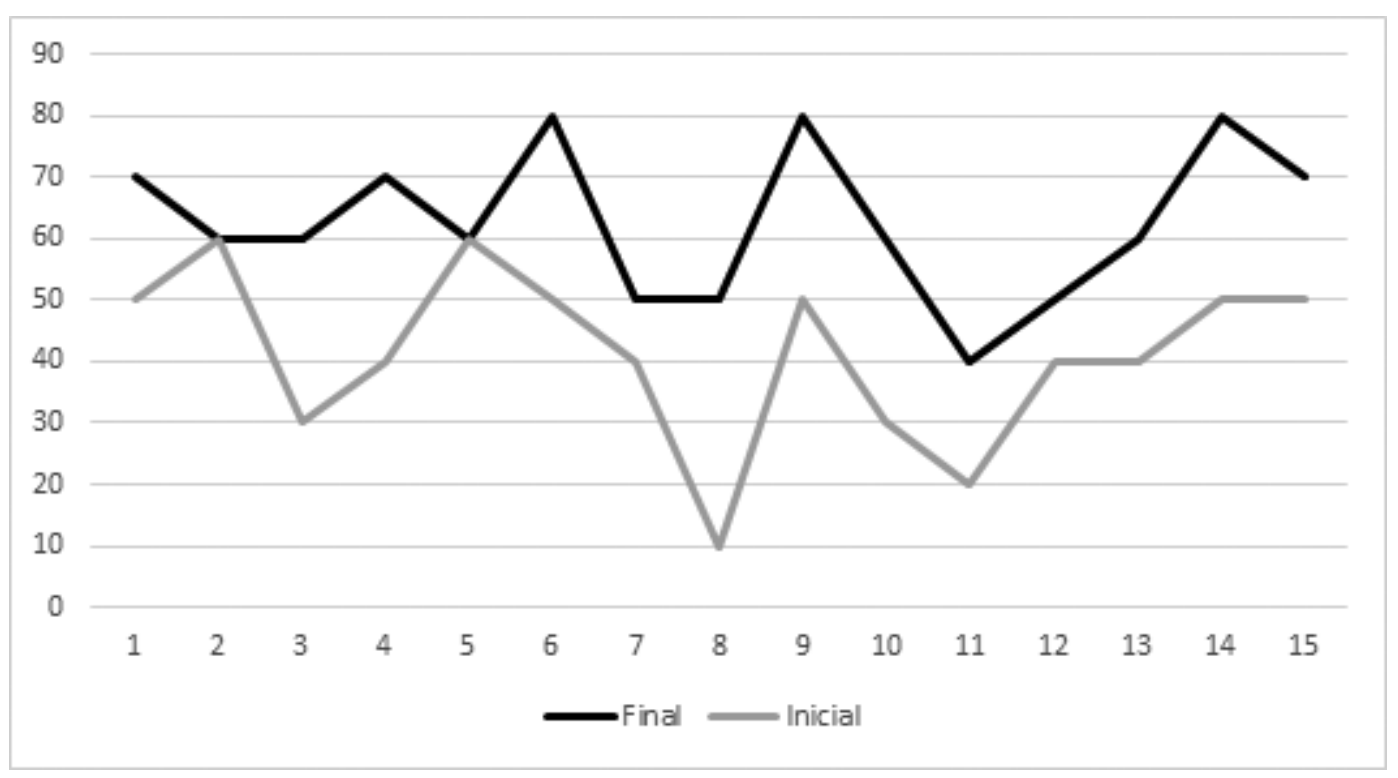

Figura 4. Resultados del Grupo Piloto en las Pruebas diagnóstica y final.

La observación de las clases durante la implementación del EVA, indica un alto grado de motivación en los estudiantes; aproximadamente entre el 95 al 98\% de ellos, dependiendo de la sesión, se mostraron dispuestos a cambiar la clase magistral por el desarrollo de las actividades planteadas en el EVA. Los estudiantes llegaban temprano a clase, realizaban preguntas e incluso buscaron actividades semejantes para presentarlas a sus compañeros y al profesor.

La competencia de interpretación y solución de problemas se desarrolló también mediante la estructuración de instrucciones para la solución autónoma de situaciones problémicas, incentivando la participación e incluso investigación de los estudiantes, aspecto no esperado en el estudio. Esto se hizo a partir de unos conocimientos previos, diagnosticados bajo la intervención del docente. Otro aspecto relevante fue que el rol de estudiantes y profesor quedaron claros: los primeros asumiendo un compromiso con su aprendizaje; y el segundo, moderando su rol, consciente de las responsabilidades que debía otorgarles.
Lo anterior sugiere que la actitud de los estudiantes hacia las prácticas tradicionales en el aula, como: la clase magistral, dictados, transcripción de textos en el tablero, entre otros, es de rechazo. Asimismo, las prácticas innovadoras que integran recursos TIC, motivan al estudiante no solo a trabajar en los contenidos y temáticas propuestas, sino también a la participación, atención, puntualidad, concentración y disposición a la clase, como ha sido reportado por otros investigadores (Claro, 2010; Tangarife-Chalarca, 2013; Peláez \& Osorio, 2015).

Los puntajes obtenidos en esta etapa pueden abordarse de dos formas. La primera, reconociendo algunos aspectos a mejorar en el proceso de enseñanza aprendizaje; esto debido a que fueron varios los estudiantes que reprobaron o aprobaron con puntajes regulares. La segunda, considerando el avance de la mayoría de los estudiantes e incluso los resultados sobresalientes de algunos de ellos. Este último aspecto, por encontrarse más acentuado, se considera la mejor manera de interpretar el proceso. 
En otras palabras, los resultados apuntan a que la integración de las TIC si fortaleció la competencia de interpretación y solución de problemas en los estudiantes de grado décimo. En contraste, el grupo que no utilizó el EVA, aunque vio los mismos contenidos, siempre a tiempo por no necesitar de un aula especial, no alcanzó los resultados mínimos que pudieran demostrar un fortalecimiento de sus competencias de interpretación y solución de problemas.

Entre las limitaciones del estudio cabe resaltar: la disponibilidad del espacio para trabajar el EVA era limitada, por tanto el proceso se llevó a cabo de manera discontínua; el tiempo de implementación no fue el adecuado, solo 60 minutos semanales cuando era posible acceder al aula de informática; además, la situación socioeconómica de muchos estudiantes no permite que estos adelanten en casa las actividades, por falta de dispositivos y conectividad. Estas dificultades son frecuentes a la hora de implementar las TIC, especialmente en instituciones educativas de carácter público, debido a la brecha digital existente en Colombia (García-Amaya et al., 2017).

\section{Conclusiones}

El objetivo de esta investigación fue determinar cuál es el impacto de un Entorno Virtual de Aprendizaje, EVA, en el proceso de enseñanza aprendizaje de la competencia de interpretación y solución de problemas, en estudiantes de grado décimo de una institución educativa de Cali, Colombia.

Los resultados de las pruebas diagnóstica y final, permiten afirmar que el EVA tuvo un impacto positivo en el desarrollo de la competencia por parte de los estudiantes. No obstante, se reconoce la necesidad de fortalecer algunos aspectos en el proceso de enseñanza aprendizaje, pues los resultados no fueron plenamente favorables. Esto se explica en parte por el poco tiempo de implementación, tres meses en este caso, unido a las dificultades de acceso al aula de informática y a la inestabilidad de la conexión a internet.
En cuanto a los estudiantes, la investigación revela que su poco interés en el proceso de enseñanza aprendizaje depende en muchas ocasiones de la falta de nuevas iniciativas para el trabajo en el aula. Sin embargo, ante nuevas propuestas se presentan participativos y comprometidos, en este caso integrando las TIC como elemento diferenciador. Asimismo, los EVA no son la única herramienta que permiten tal escenario, sino tan solo una entre muchas alternativas en la estimulación, tanto de las competencias mencionadas, como del proceso de enseñanza en general.

Lo anterior indica que los estudiantes tienen necesidad de cambio en cuanto a los modelos pedagógicos tradicionales, requiriendo nuevas didácticas en los encuentros de clase, para construir un proceso de enseñanza aprendizaje más dinámico y productivo. El papel de los docentes debería ser el de liderar la incorporación de nuevas estrategias formativas al aula, lo cual permitiría mejorar en gran medida la educación actual de nuestra ciudad y, por qué no, de nuestro país.

En síntesis, la interpretación y solución de problemas es una de las competencias básicas que las personas, tanto en el nivel académico como en la vida cotidiana deben apropiar. Esto indica la importancia de fomentar su enseñanza particularmente en el ámbito escolar, ya que es allí donde los ciudadanos adquieren las competencias básicas para el resto de su vida. En este sentido, es necesario vincular nuevas herramientas al aula, como el EVA de la presente investigación, las cuales generan una mayor motivación y mejoran la adquisición de competencias en los estudiantes.

\section{Referencias}

Aguirre, H. (2015). Experiencia educativa con Kodu en El Salvador. Diá-Logos, 0 (12), 67-75. doi: http:// dx.doi.org/10.5377/dialogos.v0i12.2194

Aldana-Bermúdez, E., \& López-Mesa, J. (2016). Matemáticas para la diversidad: un estudio histórico, epistemológico, didáctico y cognitivo sobre 
perímetro y área. Revista de Investigación, Desarrollo e Innovación, 7 (1), 77-92. doi: http://dx.doi. org/10.19053/20278306.v7.n1.2016.5602

Almeyda-Hidalgo, L. (2016). Arrojados en la acción: Aprender a enseñar en la experiencia de práctica profesional. Estudios pedagógicos (Valdivia), 42 (4), 11-30. doi: https://dx.doi.org/10.4067/S071807052016000500002

Álvarez, G., \& Taboada, M. (2016). Propuestas didácticas mediadas por tecnologías digitales para el desarrollo de competencias de lectura y escritura académicas. Revista Guillermo de Ockham, 14(2),8391. doi:http://dx.doi.org/10.21500/22563202.2336

Astudillo, G. J., Sanz, C., \&Willging, P. A. (2012). Análisis de Compatibilidad entre Objetos de Aprendizaje basados en SCORM y LMS de Código Abierto. Conferencias LACLO, 3 (1).

Avella-lbáñez, C. P., Sandoval-Valero, E. M., \& Montañez-Torres, C. (2017). Selección de herramientas web para la creación de actividades de aprendizaje en Cibermutua. Revista de investigación, Desarrollo e Innovación, 8 (1), 107-120. doi: 10.19053/20278306.v8.n1.2017.7372

Barrera-Mesa, M., Fernández-Morales, F. H., \& Duarte, J. E. (2017a). Aprendizaje basado en proyectos colaborativos mediados por TIC para el desarrollo de competencias en estadística. Saber, Ciencia y Libertad, 12 (2), 220-232. Recuperado de: http:// www.sabercienciaylibertad.org/ojs/index.php/ scyl/article/view/247

Barrera-Mesa, C. E., Fernández-Morales, F. H., \& Duarte, J. E. (2017b). Diseño de un ambiente de aprendizaje mediado por TIC para la enseñanza de operadores mecánicos orientado al grado séptimo de la educación básica, en el Colegio Boyacá de Duitama. Revista Colombiana de Tecnologías de Avanzada, 2 (30).
Belloch, C. (2011). Diseño instruccional. Valencia, España: Universidad de Valencia. Recuperado de: http://www.uv.es/bellochc/pedagogia/EVA4.pdf

Berger, C., \& Kam, R. (2006). Definitions of Instructional Design. Adapted from Training and Instructional Design. Applied Research Laboratory, Penn State University. Recuperado de: http://www. umich.edu/ ed626/define.html

Bernal-Pinzón, M.L.(2017) ¿quéescriben los niños?, una mirada desde el modelo escuela nueva. Revista de investigación, Desarrollo e innovación, 7 (2), 255-268. doi: https://doi.org/10.19053/20278306. v7.n2.2017.6069

Blanco-Sánchez, M. I. (2012). Recursos didácticos para fortalecer la enseñanza-aprendizaje de la economía. Aplicación a la Unidad de Trabajo "Participación de los trabajadores en la empresa". Valladolid, España: Universidad de Valladolid.

Buitrago-Guzmán, S. (2014). Relación entre la convergencia de medios y la experiencia de usuario. Dos iniciativas creativas en Colombia. Revista De Investigación, Desarrollo E Innovación, 4 (2), 79-86. doi: http://dx.doi.org/10.19053/20278306.2958

Castro-Galeano, J. C., Pinto-Salamanca, M. L., \& Amaya-Quitián, M. F. (2014). Diseño y construcción de una Bobina Tesla de 1680 W, para la enseñanza de conceptos básicos en sistemas eléctricos de potencia. Revista de Investigación, Desarrollo e Innovación, 5 (1), 66-74. https://doi. org/10.19053/20278306.3142

Claro, M. (2010). Impacto de las TIC en los aprendizajes de los estudiantes: estado del arte. Comisión Económica para América Latina y el Caribe (CEPAL). Recuperado de: http://repositorio.cepal.org/bitstream/handle/11362/3781/lcw339.pdf?sequen$\mathrm{ce}=1$

Contreras-Chinchilla, L., \& Escalona-Ustariz, A. (2014). Web 2.0 como apoyo al aprendizaje de 
nuevas tecnologías en la comunidad académica: caso Universidad Popular del Cesar. Ingeniare, 9 (16), 47-62. Recuperado de: http://www.unilibrebaq.edu.co/ojsinvestigacion/index.php/ingeniare/article/view/404

Cook, D., \& Cambell, D. T. (1979). Quasi-experimentation: design \& analysis issues or field settings. Boston, USA: Houghton Mifflin Company.

Fernández, F. H., \& Duarte, J. E. (2013). El aprendizaje basado en problemas como estrategia para el desarrollo de competencias específicas en estudiantes de ingeniería. Formación universitaria, 6 (5), 29-38. Doi: https://dx.doi.org/10.4067/S07185006201300050000

García-Amaya, R. A., Fernández-Morales, F. H., \& Duarte, J. E. (2017). Modelo de integración de las TIC en instituciones educativas con características rurales. Revista Espacios, 38 (50), 26. Recuperado de: http://www.revistaespacios.com/ a17v38n50/17385026.html

García-Quiroga, B., Coronado, A., \& Giraldo-Ospina, A. (2017). Implementación de un modelo teórico a Priori de competencia matemática asociado al aprendizaje de un objeto matemático. Revista de Investigación, Desarrollo e Innovación, 7 (2), 301-315. doi:https://doi.org/10.19053/20278306. v7.n2.2017.6072

Hernández-Suárez, C., Prada-Núñez, R., \& Gamboa-Suárez, A. (2017). Conocimiento y uso del lenguaje matemático en la formación inicial de docentes en matemáticas. Revista de Investigación, Desarrollo e Innovación, 7 (2), 287-299. doi: https:// doi.org/10.19053/20278306.v7.n2.2017.6071

ICFES (2016a). Sistema nacional de evaluación estandarizada de la educación. Lineamientos generales para la presentación del examen de estado Saber $11^{\circ}$. 3ra edición. Bogotá. Colombia.
ICFES (2016b). Saber $3^{\circ}, 5^{\circ}$ y $9^{\circ}$ Lineamientos para las aplicaciones muestral y censal 2016. Bogotá. Colombia.

ICFES (2016c). Saber $3^{\circ}, 5^{\circ}$ y $9^{\circ}$ Cuadernillo de prueba. Primera edición. Matemáticas grado $9^{\circ}$. Bogotá. Colombia.

Jaramillo-Arcila, J. J., \& Quintero-Calvache, D. M. (2014). Desarrollo de un ambiente virtual de aprendizaje fundamentado en la lúdica que estimule el pensamiento aleatorio en los estudiantes de grado cuarto y quinto de primaria de la institución educativa el Hormiguero (Tesis de Maestría). Cali: Colombia: Universidad Libre.

León-Medina, J. X., \& Torres-Barahona, E. A. (2016). Herramienta para el diseño de sistemas de posicionamiento tridimensional usados en fabricación digital. Revista de Investigación, Desarrollo e Innovación, 6 (2), 155-167. doi: http://doi. org/10.19053/20278306.4603

Medina-Rojas, F., Nuñez-Santa, J., Sánchez-Medina, I., \& Cabrera-Medina, J. (2017). Implementación del ABP, PBL y método SCRUM en cursos académicos para desarrollar sistemas informáticos enfocados en fortalecer la región. Revista Educación en Ingeniería, 12 (24), 52-57. doi:http://dx.doi. org/10.26507/rei.v12n24.758

Ministerio de Educación Nacional, MEN. (2006). Estándares Básicos de Competencias en Matemáticas. Recuperado de: http://www.mineducacion.gov. co/1621/articles-116042_archivo_pdf2.pdf

Mishra, P., \& Koheler, M. J. (2006). Technological Pedagogical Content Knowledge: A framework for teacher knowledge. Teachers College Record, 108 (6), 1017-1054. doi: 10.1111/j.14679620.2006.00684.x.

Parada-Hernández, A., \& Suárez-Aguilar, Z. E. (2014). Influencia de las Tecnologías de la Información y la Comunicación en la apropiación de 
conceptos de electrónica análoga, en estudiantes de grado séptimo de educación básica. Revista de Investigación, Desarrollo e Innovación, 5 (1), 20-31. doi: https://doi.org/10.19053/20278306.3137

Parra-León, L. F., Duarte, J. E., \& Fernández-Morales, F. H. (2014). Propuesta didáctica para la enseñanza de circuitos eléctricos básicos. Revista de Investigación, Desarrollo e Innovación, 4 (2), 138-147. doi: http://doi.org/10.19053/20278306.2891

Peláez, L. E., \& Osorio, B. E. (2015). Medición del nivel de aprendizaje con dos escenarios de formación: uno tradicional y otro con TIC. Entre Ciencia e Ingeniería, 9 (18), 59-66. Recuperado de: http:// www.scielo.org.co/scielo.php?script=sci_arttext\&pid=S1909-83672015000200008\&lng=es\&tIn$\mathrm{g}=\mathrm{es}$.

Pinto-Salamanca, M. L., Sofrony-Esmeral, J. I., \& Jiménez, D. F. (2015). Detección de colisiones con librerías V-Collide y PhysX para interacción virtual con interfaces hápticas. Revista de Investigación, Desarrollo e Innovación, 5 (2), 119-128. doi: $10.19053 / 20278306.3721$

Quiroga-Parra, D. J., Torrent-Sellens, J., \& Murcia-Zorrilla, C. P. (2017). Usos de las TIC en América Latina: una caracterización. Ingeniare. Revista chilena de ingeniería, 25 (2), 289-305. doi: https:// dx.doi.org/10.4067/S0718-33052017000200289

Ramírez-Hurtado, A. L., Gil-Monsalve, J., Medina-Barreto, M. H., \& Cruz-Muñoz, B. (2016). Implementación en entorno Labview de un sistema multifuncional de medidas magnetoópticas y magnetoeléctricas para caracterización de materiales. BISTUA Revista de la Facultad de Ciencias Básicas, 14 (2), 116-125. doi: https://doi. org/10.24054/01204211.v2.n2.2016.2188

Rico-González, C. A. (2011). Diseño y aplicación de ambiente virtual de aprendizaje en el proceso de enseñanza-aprendizaje de la física en el grado décimo de la IE Alfonso López Pumarejo de la ciudad de Pal- mira (Tesis Doctoral). Palmira, Colombia: Universidad Nacional de Colombia.

Riveros-Hernández, D., Nausan-García, D., García-Miranda, D., \& Palacios-Osma, J. (2017). Desarrollo de un entorno virtual para la simulación de maniobras eléctricas en subestaciones: un caso práctico. Ingeniería solidaria, 13 (22). doi:https:// doi.org/10.16925/in.v13i22.1752

Rodríguez-Cepeda, R. (2016). Aprendizaje de conceptos químicos: una visión desde los trabajos prácticos y los estilos de aprendizaje. Revista de Investigación, Desarrollo e Innovación, 7 (1), 63-76. doi: https://doi.org/10.19053/20278306. v7.n1.2016.4403

Rosas, J. (2015). Asistencia técnica a la Institución Santo Tomás. Gestión de la evaluación para la calidad del servicio educativo. Secretaría de Educación Municipal de Santiago de Cali. Cali, Colombia.

Salcedo-Ramírez, R. Y., Fernández-Morales, F. H., \& Duarte, J. E. (2017). Unidad didáctica para la enseñanza de probabilidad mediada por un OVA, orientada a un colegio rural del municipio de Paipa. Revista Colombiana de Tecnologías de Avanzada, 2 (30).

Salinas, M. (2011). Entornos virtuales de aprendizaje en la escuela: tipos, modelo didáctico y rol del docente. Argentina: Pontificia Universidad Católica Argentina.

Tangarife-Chalarca, D. (2013). Desarrollo de una aplicación web para el montaje de una mesa quirúrgica en el área de traumatología. Revista de Investigación, Desarrollo e Innovación, 4 (1), 32-44. doi: https://doi.org/10.19053/20278306.2124

Valdelamar-Zapata, J. A., Ramírez-Cruz, Y. L., Rodríguez-Rivera, P. D., \& Morales-Rubiano, M. E. (2015). Capacidad innovadora: cómo fomentarla, según docentes de Ciencias Económicas e Ingeniería de la UMNG. Revista de Investigación, De- 
sarrollo e Innovación, 6 (1), 7-14. doi: http://doi. org/10.19053/20278306.3454

Valencia-Serrano, M., \& Caicedo-Tamayo, A. M. (2017). Diseño de tareas apoyadas en TIC para promover aprendizaje autorregulado. Pensamiento Psicológico, 15 (2), 15-28. doi: https://dx.doi. org/10.11144/Javerianacali.PPSI15-2.dtat 